\title{
INVERSE-PRODUCING EXTENSIONS OF NORMED ALGEBRAS
}

\author{
BY \\ RICHARD ARENS
}

1. Introduction. The first result presented here is that an element $c$ of a commutative normed algebra $A$ has an inverse in some norm-preserving extension $B$ ("superalgebra") of $A$ if and only if $c a_{n} \rightarrow 0$ implies $a_{n} \rightarrow 0$ for every sequence in $A$, i.e., $c$ is not a topological zero-divisor. The method may be described as consisting in constructing the algebra $A(x)$ of polynomials with coefficients in $A$, and then dividing out the ideal generated by the (first degree!) polynomial $1-c x$. The resulting algebra $B$ is always of infinite degree over $A$, except when $c$ already has an inverse in $A$.

In the classical applications of this method (usually, to fields) the coefficient of the highest power may be taken as 1 , and that sort of polynomial extension goes over in manageable fashion to normed algebras [see bibliography: Hoffman and Arens]. However, the present construction is nontrivial only because $c$ has no inverse (and effective only because it is not a topological zero-divisor). Most of our space is taken up with quantitative refinements, with systems of elements, and finally with a result on semi-simplicity, interesting perhaps mainly in view of the complexity of our proof. The question (unsolved at present) is whether, when $A$ is semi-simple, it can be arranged that $B$ is semi-simple (if it can be arranged, then it can be done by our method (see 3.71)). We are able to answer affirmatively only in the case where $c$ generates $A$ over the scalars (or some other similarly-behaving subalgebra).

We remark that all problems treated here are trivial when $A$ has the sup norm, i.e., $\|a\|=\|\hat{a}\|_{\infty}$ [see Loomis]: the algebra of all bounded (complex valued) functions on the Shilov boundary provides an extension solving all reasonable problems.

C. E. Rickart has exhibited a technique for simultaneously advancing to regularity a certain class of elements. This class is more or less the obvious one for his technique-reminiscent of the making a field out of an integral domain. Our individual- (or finitely many) element-method takes care of some elements not in Rickart's class.

Some unsolved problems already familiar to people in this field are mentioned in $\$ 7$.

2. Commutativity, and the unit. We deal only with commutative normed algebras over the complex numbers. But the main thing to be kept in mind,

Presented to the Society, August 21, 1956 under the title Removing ideals by Banach algebra extension; received by the editors January 12, 1957. 
because we do not repeat it, is that the algebra $A$ is supposed to have a unit element 1 , whose norm is 1 .

An appendix deals with the case of no unit, for Banach (i.e., complete) normed algebras.

3. Supplying inverses. This section deals with the establishment and elaboration of the following proposition, which may be paraphrased by saying that an element has an inverse in some extension algebra precisely if it is not a topological divisor of zero.

3.1. TheOREM. Let $c$ be an element of a commutative normed algebra (see §2) $A$. Then $c$ has an inverse in some superalgebra $B$ of $A$ if and only if

$$
\inf _{\|a\| \geqq 1}\|c a\|>0 \text {. }
$$

Specifically, $c$ has an inverse of norm not exceeding $t_{0}$ in some superalgebra $B$ of $A$ if and only if, for all $a$ in $A$,

$$
t_{0}\|c a\| \geqq\|a\| \text {. }
$$

Proof. If $c x=1$ for some $x$ in some superalgebra $B$ of $A$, and $a \in A$ then $a=\operatorname{cax},\|a\| \leqq\|c a\|\|x\|$, showing the necessity of 3.12 . We proceed to show its sufficiency.

Select any real non-negative $t$. Let $A(x ; t)$ be the class of formal power $\operatorname{series}\left({ }^{1}\right)$

$$
f(x)=a_{0}+a_{1} x+a_{2} x^{2}+\cdots
$$

in which $a_{n}, a_{1}, a_{2}, \cdots$ are elements of $A$ such that

$$
\|f(x)\|=\sum_{n=0}^{\infty}\left\|a_{n}\right\| t^{n}<\infty .
$$

It is not hard to see that $A(x ; t)$ is a normed algebra. It contains $A$ as a subalgebra. Let $J$ be the smallest closed ideal in $A(x ; t)$ containing $1-c x$; and let

$$
A(t)=A(x ; t) \bmod J .
$$

It is easily verified that with the canonical norm (for the canonical image of $f(x))$

$$
\|f(x)-J\|=\inf _{j(x) \in J}\|f(x)-j(x)\|
$$

$A(t)$ emerges as a normed algebra, and in this algebra, the (canonical image of the) element $c$ has an inverse, the (canonical image of the) element $x$.

(1) One could limit himself to polynomials here and then the final $B$ would be a normed algebra, but perhaps incomplete. The closure would then serve the purpose of 3.1 . But as it stands, $A(x, t)$ is complete when $A$ is. 
Clearly $\|f(x)-J\| \leqq\|f(x)\|$. Unless precautions are taken, $A$ may reappear with diminished norms in $A(t)$, and this would not yield the result desired in 3.1. The following (whose statement and proof we insert in the body of the proof, here, of 3.1) will show that this danger is avoided by taking $t \geqq t_{0}$.

3.2. Lemma. Let $t_{0}\|c a\| \geqq\|a\|$ for all $a$ in $A$. Let $a$ be selected in $A$, and $f(x)=a_{0}+a_{1} x+\cdots$ in $A(x, t)$. Then $\left({ }^{2}\right)$ (in $\left.A(x, t)\right)$

$$
\|a-(1-c x) f(x)\| \geqq\|a\|+\left(t t_{0}^{-1}-1\right)\|f(x)\| \text {. }
$$

Proof.

$$
g(x)=a-(1-c x) f(x)=a-a_{0}+\left(c a_{0}-a_{1}\right) x+\left(c a_{1}-a_{2}\right) x^{2}+\cdots
$$

and so

$$
\|g(x)\|=\left\|a-a_{0}\right\|+\left\|c a_{0}-a_{1}\right\| t+\left\|c a_{1}-a_{2}\right\| t^{2}+\cdots .
$$

The condition on $c$, and the triangle inequality readily yield (for all $a, b$ in $A$ )

$$
\|c a-b\| \geqq u\|a\|-\|\dot{b}\| \text {. }
$$

Using 3.22 and also $\left\|a-a_{0}\right\| \geqq\|a\|-\left\|a_{0}\right\|$, we obtain

$$
\begin{aligned}
\|g(x)\| & \geqq\|a\|-\left\|a_{0}\right\|+\left(u\left\|a_{0}\right\|-\left\|a_{1}\right\|\right) t+\cdots \\
& =\|a\|+(t u-1)\left(\left\|a_{0}\right\|+\left\|a_{1}\right\| t+\cdots\right),
\end{aligned}
$$

which is precisely 3.21 . This establishes 3.2 .

Continuing with 3.1 , we select $t=t_{0}$.

How big is the norm of $a$ in $A(t)$ ? By 3.21, it is not less than $\|a\|$. Obviously $\|a-J\| \leqq\|a\|$. Therefore $\|a-J\|=\|a\|$. We discover that $A$ is isometrically imbedded in $A(t)$. The norm of $x$ in $A(x ; t)$ is $\left(^{3}\right) t$; it cannot be greater in $A(t)$. This suffices to prove 3.1.

3.2 can be used also to establish the following:

3.3. Theorem. When $t>t_{0}$, the principal ideal $\{f(x)-c x f(x)\}$ is (already) closed, and the operator $T, T f(x)=(1-c x) f(x)$, has an inverse of bound not greater than $\left(t t_{0}^{-1}-1\right)^{-1}$. When $t=t_{0}$, the ideal may not be closed (and then, of course, $T$ has no bounded inverse).

Proof. Let $a=0$ in 3.21, and observe $T f(x)=\left(t t_{0}^{-1}-1\right)(f(x))$. The statement about $t=t_{0}$ describes a classical situation. Let $A=$ complex numbers, $c=1$, then $A(x ; 1)$ is the class of absolutely convergent power series $(J$, those that vanish at 1$)$ :

$$
\sum_{n=1}^{\infty} \frac{1-x^{n}}{n^{2}}
$$

(2) $A$ is not here required to contain a 1 ; for in the expansion of $(1-c x) f(x), 1$ will not appear explicitly.

(3) If $\|1\|$ were not 1 , we would have $x=t\|1\|$. See the appendix. 
belongs to $J$, but not to $(1-x) A(x ; 1)$.

Instead of solving the equation $1-c x=0$, we can treat the equation $c_{0}+c_{1} x=0\left(c_{0}, c_{1} \in A\right)$. If this is to have a solution $(x)$ in some superalgebra with $\|x\| \leqq 1$, it is evidently necessary that

3.4

$$
\left\|c_{0} a\right\| \leqq\left\|c_{1} a\right\| \quad(\text { all } x \in A) .
$$

3.5. Theorem. This condition is also sufficient.

The proof and construction is carried out just as before ( $A$ need not have a 1 for this theorem). At the point where 3.2 intervenes, we use instead:

3.6. LEMma. If 3.4 holds, then for any $f(x) \in A(x, t)$ we have

$$
\left\|a-\left(c_{0}+c_{1} x\right) f(x)\right\| \geqq\|a\|+(t-1)\left\|c_{0} f(x)\right\| .
$$

The proof will be left to the reader.

The ideal $\left\{\left(a_{0}+a_{1} x\right) f(x)\right\}$ need not now be closed even for $t>1$, because $c_{0}$ might be a generalized zero divisor.

Polynomials equations $c_{0}+c_{1} x+\cdots+c_{k} x^{k}=0$ cannot be treated by this technique for $k>1$.

The solutions $A(t)\left(t \geqq t_{0}\right)$ provide in the following sense, the most economical solution to the problem of making an inverse for $c$.

3.7. Theorem. Let 3.12 hold. Suppose $B$ is any normed algebra containing $A$ in which $c$ has an inverse $y$ of norm $t\left(t \geqq t_{0}\right)$. Then $A(t)$ can be mapped naturally into $B$ with a homomorphism of bound not exceeding 1.

Proof. First we define $T: A(x, t) \rightarrow B$ where

$$
T(f(x))=\sum a_{n} y^{n}=b \text {. }
$$

Surely

$$
\|b\| \leqq \sum\left\|a_{n}\right\| t^{n}=\|f(x)\| \text {, so }\|T\| \leqq 1 .
$$

Now the kernel $K$ of this homomorphism includes $J$, so that we have

$$
A(t)=A(x, t) \bmod J \rightarrow A(x, t) \bmod K \rightarrow B .
$$

The first of these is of bound $\leqq 1$ since we are using canonical norms; and the second, because of a normed space fact [cf. Loomis, 7. D].

3.71. Corollary. If any such $B$ is semi-simple, then so are all the $A(t)$ $\left(t \geqq t_{0}\right)$.

We now turn to the consideration of several elements at once. Perhaps the most useful result is obtained by reducing the problem to one element.

3.8. Theorem. Let $c_{1}, \cdots, c_{n}$ be finitely many elements of a commutative normed algebra $A$, and suppose there are numbers $t_{1}, \cdots, t_{n}$ such that 


$$
t_{k}\left\|c_{k} a\right\| \geqq\|a\| \quad(\text { all } a \in A ; \text { all } k) .
$$

Then (and only then) there is a superalgebra $B$ in which all these elements have inverses.

To obtain $B$ we take $A(t)$ with $t=t_{1} t_{2} \cdots t_{n}$ and $c=c_{1} c_{2} \cdots c_{n}$, observing that $t\|c a\| \geqq\|a\|$ for all $a$. Thus $c$ has an inverse in $B$, and hence all of the $c_{k}$ do, too.

In discussing the situation we will suppose all the $t_{k}$ are 1 . Nothing of interest is lost by that. Now the trouble with 3.8 is this: whereas by individual constructions each $c_{k}$ receives an inverse of norm 1 (or less), the most that can be said here is that (for example)

$$
\left\|c_{1}^{-1}\right\| \leqq\left\|c_{2} \cdots c_{n}\right\|
$$

so that (unless say all $\left\|c_{k}\right\|=1$ : "isometric elements" (cf. Rickart)) conceivably the norms could be greater than 1 . Consider the following infinite family of conditions on a subset $H$ of a commutative Banach algebra $A$ :

3.9. Let $p\left(c_{1}, \cdots, c_{n}\right)$ be a polynomial in the $c_{i} \in H$, with coefficients in $A$; and let the degree in $c_{k}$ be less than $m_{k}$ for each $k$. Suppose, for some $a \in A$

$$
a_{1}^{m_{1}} c_{2}^{m_{2}} \cdots c_{n}^{m_{n}}=p\left(c_{1}, \cdots, c_{n}\right) \text {. }
$$

Then

$$
\|a\| \leqq \sum\|d\|
$$

where this sum runs over all the coefficients of $p$.

N.B. This is not a theorem, but a condition on a subset $H$ of $A$. The "trouble" with 3.8 is that I do not know whether the condition

$$
\|c a\| \geqq\|a\| \text { for all } a \in A \text { and all } c \in H
$$

implies all of 3.9 (of which it is a special case). The "trouble" is no more than that, because the following is true.

3.93. Theorem. Let $H$ be a subset of a commutative Banach algebra $A$. Then a necessary and sufficient condition that there is a superalgebra $B$ of $A$ in which all elements of $H$ have inverses of bound not exceeding 1 is that 3.9 hold.

Proof. We consider first the necessity. Suppose 3.91 holds. Pass to a superalgebra $B$ and let $x_{c}$ be the inverse of $c \in H\left(\left\|x_{c}\right\| \leqq 1\right)$. Multiply 3.91 on both sides by $x_{1}^{m_{1}} \cdots x_{n}^{m_{n}}$. Then all the indeterminates $c_{i}$ of $p$ will disappear, leaving just the old coefficients multiplied by a few $x$ 's. Thus we have 3.92. We might point out that several conclusions can sometimes be drawn from an equation like 3.91 (when 3.9 is assumed). Let $n=1$, and suppose

$$
a c^{3}=b c^{4}+d c .
$$

Here we cannot assert $\|a\| \leqq\|b\|+\|d\|$ because 4 exceeds 3 . However, we can 
regard $b c^{2}$ as the coefficient of $c^{2}$ and conclude $\|a\| \leqq\left\|b c^{2}\right\|+\|d\|$. It is not hard to see that even $\|a\| \leqq\|b c\|+\|d\|$ can be concluded.

We now pass to the construction which shows the sufficiency.

This time we make a polynomial algebra first, instead of a complete one as in 3.1. Make a family of indeterminates $\left\{x_{c}\right\}$, one for each $c$ in $H$. Let $A(x)$ be the algebra of polynomials in these $x_{c}$, coefficients in $A$. For $p(x)$ $\in A(x)$ let

$$
\|p(x)\|=\sum\|a\|
$$

$a$ running through all coefficients in $p(x)$. Let $J$ be the ideal generated by the $1-c x_{c}, c \in H$. We pass to the algebra $A(x) \bmod J$ and introduce the canonical norm (and later complete. This will give $B$.). In order to ensure that norms in $A$ are not diminished in this process it suffices to show that if

$$
g\left(x_{1} \cdots x_{n}\right)=a-\sum_{k=1}^{n}\left(1-c_{k} x_{k}\right) f_{k}\left(x_{1} \cdots x_{n}\right)
$$

(where $x_{k}$ is written for $x_{c}$ when $c=c_{k} \in H$ ), then $\|g(x)\| \geqq\|a\|$. To see that this is true we now multiply 3.94 by a power product $c_{1}^{m_{1}} \cdots c_{n}^{m_{n}}$ with $m_{k}$ greater than every exponent of $x_{k}$ appearing. Then we have the identity

$$
c_{1}^{m_{1}} \cdots c_{n}^{m_{n}} g(x)=a c_{1}^{m_{1}} \cdots c_{n}^{m_{n}}-s(x),
$$

and this is to be considered as multiplied out so that each $x_{k}$ appears only in the combination $c_{k} x_{k}$. We then set each $c_{k} x_{k}=1$. It results that

$$
a c_{1}^{m_{1}} \cdots c_{n}^{m_{n}}=c_{1}^{m_{1}} \cdots c_{n}^{m_{n}} g\left(1 / c_{1}, \cdots, 1 / c_{n}\right)
$$

where the right side is really a polynomial $Q\left(c_{1}, \cdots, c_{n}\right)$ whose distinct coefficients are the coefficients of $g$. By $3.9,\|a\| \leqq \sum\|d\|$ where $d$ runs over the coefficients of $Q$, but this we have just pointed out is the same as

$$
\begin{aligned}
\|a\| & \leqq \sum\|d\|(d \text { runs through the coefficients of } g) \\
& \leqq\|g(x)\| .
\end{aligned}
$$

This suffices to establish 3.93 .

Of course, if in any of these cases a complete algebra is desired, one may complete the algebra.

4. Topological zero divisors in commutative Banach algebras. It was asserted in $[1$, p. 628] that the frontier points of the set of singular elements (those with no inverse) are topological zero divisors, but the proof there suggested requires completeness. Indeed, the theorem is exact only with such a qualification, as examples (polynomial algebras) show. For definiteness, we present the proposition here, together with some quantitative refinements, involving the following concept. Given an element $c$, consider the greatest number $\xi$ for which it can be said that 
This may be called the modulus of integrity. It may be 0 , and it is 0 if and only if $c$ is a topological zero divisor. If $c^{-1}$ exists then $\xi\left\|c^{-1}\right\|=1$. Moreover, it is not hard to prove that

$$
\xi_{c+a} \geqq \xi_{c}-\|a\|, \quad \xi_{a c} \geqq \xi_{a} \xi_{c},
$$

which represents a generalization of the well-known relation

$$
\left\|(c+a)^{-1}\right\| \leqq \frac{\left\|c^{-1}\right\|}{1-\|a\|\left\|c^{-1}\right\|} .
$$

4.3. Theorem. A frontier point of the set $S$ of singular elements is a topological zero divisor. If $c \in S$ and $\|a\| \leqq \xi_{c}$ then $c-a$ is also singular. In particular (if $c \in S$ then) the spectrum of $c$ covers the disc of radius $\xi_{c}$ centered at origin.

Proof. Let $r_{n} \rightarrow c$, where $a_{n} r_{n}=1$. Then $a_{n} c=1+a_{n}\left(c-r_{n}\right)$, and since this cannot have an inverse, we must have ( $A$ being complete)

$$
1 \leqq\left\|a_{n}\left(c-r_{n}\right)\right\| \leqq\left\|a_{n}\right\|\left\|c-r_{n}\right\| .
$$

Therefore $a_{n}\left\|a_{n}\right\|^{-1} c$ tends to 0 , which brands $c$ as a topological zero divisor [cf. Shilov].

Suppose $0<\|a\| \leqq \xi_{c}$, but imagine $c-a$ to have an inverse. Then there must be a real scalar $r, 0 \leqq r<1$ such that $b=c-r a$ is a frontier point of $S$, whence $\xi_{b}=0$. But by 4.2 we should have $\xi_{b} \geqq \xi_{c}-r\|a\| \geqq\|a\|(1-r)>0$. In particular, for $a=\lambda$ (we denote scalar multiples of the 1 in $A$ by the same symbol as the scalar itself), we obtain: $c-\lambda$ has no inverse unless $|\lambda|>\xi_{c}$.

This latter result follows also from 3.1, as frontier points of the spectrum can never be lost upon extension.

5. General remarks on the semi-simplicity. We shall consider the algebra $A(t)$ (see 3.15) where $t \geqq t_{0}$. We shall suppose here that $A$ has a 1 , so that the space $\Delta(A)$ of (continuous) complex valued, nonzero, homomorphisms of $A$ forms a compact set in its natural $\left({ }^{4}\right)$ (the weak) topology.

5.1. Theorem. $\Delta A(x, t)$ and $\Delta(A) \times\{\lambda:|\lambda| \leqq t\}$ are naturally homeomorphic.

The correspondence we have in mind is that in which a homomorphism $\zeta$ of $A(x, t)$ is associated with $(\eta, \lambda)$ where $\eta$ is $\zeta$ cut down to $A$, and $\lambda=\zeta(x)$. The proof of 5.1 may be omitted here.

5.2. THEOREM. $\Delta(A(t))$ is naturally homeomorphic with that subset of $\Delta(A) \times\{\lambda:|\lambda| \leqq t\}$ consisting of the $(\eta, \lambda)$ such that $\eta(c) \lambda=1$, and that in turn is naturally homeomorphic to the part of $\Delta(A)$ consisting of those $\eta$ such that $|\eta(c)| \geqq t^{-1}$.

(4) The Stone topology is the natural topology for the space of maximal ideals, however. 
Proof. The first assertion comes from the fact that the $\Delta$ of quotient ring corresponds to the hull of the ideal, in this case generated by $c x-1$. The second assertion follows from the fact that on that hull, the $\lambda$ is determined by the $\eta$ alone.

5.21. Corollary. $A(t)$ is semi-simple if and only if whenever

$$
\sum_{n=0}^{\infty}\left\|a_{n}\right\| l^{n}<\infty \quad\left(a_{n} \in A\right)
$$

and

$$
\sum \eta\left(a_{n}\right) \eta(c)^{-n}=0 \text { for all } \eta \in \Delta(A) \text { such that }|\eta(c)| \geqq t^{-1}
$$

then for every positive $\epsilon$ there is a sequence $b_{0}, b_{1}, b_{2}, \cdots$ in $A$ such that $\sum\left\|b_{n}\right\| t^{n}<\infty$ and (see 3.14)

$$
\left\|\sum a_{n} x^{n}-(1-c x) \sum b_{n} x^{n}\right\|<\epsilon .
$$

The condition here just says that if $f(x)=\sum a_{n} x^{n}$ vanishes on the hull of $(1-c x)$, then it lies in the closure of the ideal generated by $1-c x$. Thus the truth of 5.21 is obvious. Those $f(x)$ for which 5.23 does hold, but 5.24 does not hold (for some $\epsilon$ ) are in (or, rather, pass into) the radical of $A(t)$. It is interesting, although we have not been able to exploit it and obtain a general result, that if in 5.21, the element $f(x)=a_{0}+a_{1} x+\cdots$ involved is a polynomial, and satisfies 5.23, and $A$ is semi-simple, then $f(x)$ belongs to the ideal $(1-c x)$ (not merely the closure). This can be amplified somewhat, but still not very usefully, as follows:

5.3. Theorem. If 5.23 holds for a polynomial $f(x)=a_{0}+a_{1} x+\cdots+a_{N} x^{N}$ then $f(x)=g(x)(1-c x)+r x^{N}$ where $g(x)$ is another polynomial and $r$ is in the radical of $A$.

Proof. Supposing first that $f(x)$ is not merely a polynomial, we write down an identity:

$$
f(x)=(1-c x) g_{N}(x)+r_{N} x^{N}+\sum_{n>N} a_{n} x^{n}
$$

where

$$
r_{N}=a_{N}+a_{N-1} c+\cdots+a_{0} c^{N}
$$

and

$$
g_{N}(x)=\sum_{n<N}\left(a_{n}+a_{n-1} c+\cdots+a_{0} c^{n}\right) x^{n} .
$$

Now back to the polynomial case. Multiplying the equation of 5.23 by $\eta\left(c^{N}\right)$ we obtain that $\eta\left(r_{N}\right)=0$ whenever $|\eta(c)| \geqq t^{-1}$. As explained above, this means that $r_{N}$ is a generalized nilpotent element of $A(t)$, and, belonging to $A$ (which is isometrically in $A(t)$ ), also of $A$. Since the remainder series in 5.31 
is zero, we have thus established 5.3. Since the norm of the remainder series tends to 0 we have also the corollary:

5.4. Corollary. $A(t)$ is semi-simple if, whenever 5.23 holds, then $\left\|r_{N}\right\| t^{N} \rightarrow 0$.

5.5. ThEOREM. If $t>t_{0}$ and $A(t)$ is semi-simple, then $\sum\left\|r_{N}\right\| t^{N}<\infty$ whenever 5.23 holds.

Proof. When 5.23 holds, the element $f(x)$ passes into the radical, and hence must be in the ideal $(1-c x)$, which is now closed (3.3). The quotient of $f(x)$ by $1-c x$ is none other than $\sum r_{N} x^{N}$, whence the theorem.

6. Algebras of 1 generator. In this section we deal entirely with a semisimple Banach algebra $A$ which is generated by an element $c$ such that

$$
\|c a\| \geqq\|a\|
$$$$
\text { (all } a \in A \text { ). }
$$

$A$ is supposed to have a $1,\|1\|=1$. We make no further assumptions about the norm.

On the basis of 6.1 we may construct $A(x, t)$ with any $t \geqq t_{0}$, where $t_{0}$ is (by 6.1 ) not greater than 1 , and finally make $A(t)$ in which this $c$ will have an inverse. Our objective is the following:

6.2. Theorem. If $t$ exceeds the spectral radius of $c$, then $A(t)$ is semi-simple.

6.3. Lemma. The algebra $A$ is (equivalent to) an algebra of continuous functions on the spectrum of $c$ (which includes the unit disc) which are holomorphic on the interior of that spectrum, in a correspondence which assigns to each polynomial in $c$ the corresponding polynomial in $z$. If $f \in A$ and $f(0)=0$ then the element $g$, where

$$
g(z)=f(z) / z
$$

belongs to $A$.

Proof. In this proof we regard $A$ as a function algebra. The functional representation is a familiar conclusion from the semi-simplicity and the fact that $c$ generates $A$. The last point is more subtle. We can find polynomials $p_{n}(z)$ such that $\left\|p_{n}(z)-f(z)\right\| \rightarrow 0$. Now $\left\|p_{n}-f\right\| \geqq|p(0)-f(0)|$ because homomorphisms are of bound 1 , and therefore

$$
\left\|z q_{n}-f\right\| \leqq 2\left\|p_{n}-f\right\|
$$

where $q_{n}=z^{-1}\left(p_{n}-p_{n}(0)\right)$. Because $q_{n}$ is a polynomial in $z$, it surely belongs to $A$.

The $z q_{n}$ form a Cauchy sequence, and so by 6.1 , the $q_{n}$ form a Cauchy sequence. Let it converge to $q$. It is easily seen that $f=z q$, q.e.d.

Let

$$
f(x)=a_{0}+a_{1} x+a_{2} x^{2}+\cdots
$$


be an element of $A(x, t)$. Then each element $a_{n}$ appearing here has a formal expansion

6.31

$$
a_{n} \sim \alpha_{n 0}+\alpha_{n 1} z+\alpha_{n 2} z^{2}+\cdots
$$

where the $\alpha_{n k}$ are complex numbers. It is clear that

$$
b_{n}=\alpha_{n 0}+\alpha_{n 1} c+\cdots+\alpha_{n n-1} c^{n-1}
$$

belongs to $A$, and hence so does $a_{n}-b_{n}$. By 6.3 we may divide out $c^{n}$, obtaining 6.33

$$
h_{n} \sim z^{-n}\left(a_{n}-b_{n}\right) \sim \alpha_{n n}+\alpha_{n n+1} z+\alpha_{n n+2} z^{2}+\cdots
$$

as an element of $A$. Concerning these elements we can say the following:

6.4. Lemma. Let $t$ exceed the spectral radius of $c$. Then

$$
\sum\left\|h_{n}\right\|<\infty \text {. }
$$

Proof. $\left\|h_{n}\right\| \leqq\left\|c^{n} h_{n}\right\| \leqq\left\|a_{n}\right\|+\left\|b_{n}\right\|$. Now $\sum\left\|a_{n}\right\|<\infty$ since the spectrum of $c$ covers the unit circle (4.3), and even $\sum\left\|a_{n}\right\| t^{n}<\infty$. In general $\left\|b_{n}\right\|$ is not of the same order of magnitude as $\left\|a_{n}\right\|$. But

$$
\alpha_{n k}=\frac{1}{2 \pi i} \int a_{n}(z) z^{-k-1} d z
$$

where we may take a contour of radius 1 about 0 , but perhaps no more. At any rate (and this does not depend on the value of $t$ ),

$$
\left|\alpha_{n k}\right| \leqq\left\|a_{n}\right\| \text {. }
$$

Now there is an $N$ depending only on $t$ such that $t^{n}>\left\|c^{n}\right\|$ for $n>N$. Hence

$$
\left\|\sum_{k>N}^{n} \alpha_{n k} c^{k}\right\|<\left\|a_{n}\right\| \sum_{k>N}^{n} t^{k}<t^{n+1}\left\|a_{n}\right\|(t-1)^{-1} .
$$

As for the earlier terms of $b_{n}$ we just say that

$$
\left\|\sum_{k \leqq N} \alpha_{n k} c^{k}\right\| \leqq\left\|a_{n}\right\|\left(1+\|c\|+\cdots+\left\|c^{N}\right\|\right) .
$$

Combining this with 6.44 gives $\sum\left\|b_{n}\right\|<\infty$; and this yields 6.41 .

The first major use of the semi-simplicity occurs now.

6.5. Lemma. Let $t$ exceed the spectral radius of $c$, and suppose 5.23 holds for $f(x)$ as in 6.31. Then

\subsection{1}

$$
\sum h_{n}=0
$$

and

6.52

$$
\left\|\sum_{n=1}^{N} h_{n}\right\| \rightarrow 0 .
$$


Proof. 6.52 follows from 6.51, of course, so we shall prove 6.51. In 6.51 the sum exists by 6.4. Moreover the representing function $h(z)=\sum h_{n}(z)$ is continuous on the spectrum of $c$ and holomorphic in the interior. Its behavior for $|z|=1$ is that of the boundary values of a function holomorphic for $|z|<1$.

Turning to another function, we observe that $\sum\left|b_{n}(z)\right|<\infty$ for $z$ in the spectrum of $c$, and so

$$
\sum\left|z^{-n} b_{n}(z)\right|<\infty \quad(|z| \geqq 1) .
$$

Hence

$$
b(z)=-\sum z^{-n} b_{n}(z)
$$

defines a function holomorphic for $|z|>1$ and continuous for $|z|=1$.

Now we apply 5.23. It tells us that

$$
\sum z^{-n} a_{n}(z)=0 \quad \text { for }|z| \geqq t^{-1} .
$$

Therefore $h(z)=b(z)$ for $|z|=1$. This enables us to define an entire function on the plane, using $h(z)$ for $|z| \leqq 1$ and $b(z)$ for $|z| \geqq 1$, which tends to 0 as $|z| \rightarrow \infty$. Such a function is, of course, necessarily 0 . Therefore $h(z)=0$, and since $A$ is semi-simple, we have established 6.51 .

6.6. Lemma. Suppose 5.23 holds, where $t>1$. Let $b_{n}\left(x^{-1}\right) x^{n}$ denote the polynomial obtained by replacing $c$ in 6.32 by $x^{-1}$ and then multiplying by $x^{n}$, i.e., 6.61

$$
b_{n}\left(x^{-1}\right) x^{n}=\alpha_{n 0} x^{n}+\alpha_{n 1} x^{n-1}+\cdots+\alpha_{n-1} x
$$

(and $\left.b_{0}\left(x^{-1}\right) x=0\right)$. Then

$$
\left\|\sum_{n=1}^{N} b_{n}\left(x^{-1}\right) x^{n}\right\| \leqq(t-1)^{-1} \sum_{n>N}\left\|a_{n}\right\| t^{n} .
$$

Proof.

$$
\sum b_{n}\left(x^{-1}\right) x^{n}=\sum_{k=1}^{N} x^{k} \sum_{n=k}^{N} \alpha_{n n-k} .
$$

By 5.23,

$$
\sum a_{n}(z) z^{-n}=0 \text { for }|z|=t^{-1} .
$$

Multiply by $z^{k-1}$, and integrate $\int \cdots d z$ over the circle of radius $t^{-1}$ about the origin. This yields (see 6.31 )

$$
\sum_{n=k}^{\infty} \alpha_{n-k}=0 .
$$

(This is one of the crucial results of the section.) Hence the coefficient of $x^{k}$ in 6.63 is equal to 


$$
\sum_{n>N} \alpha_{n n-k} .
$$

By 6.43 , its norm does not exceed $\sum_{n>N}\left\|a_{n}\right\| \equiv M$, and the norm of the corresponding term in 6.63 does not exceed $t^{k} M$. We sum over $k$, and obtain

$$
\begin{aligned}
& \text { left side of } 6.62 \leqq\left(t+\cdots+t^{N}\right) M \\
& \leqq(t-1)^{-1} t^{N+1} M \\
& \leqq(t-1)^{-1} \sum_{n>N} \| a_{n} t^{n},
\end{aligned}
$$

as was to be proved.

The last of these lemmas uses all these results.

6.7. Lemma. Let 5.23 hold, and suppose $t$ exceeds the spectral radius of $c$. Then, for every $N$ there is a polynomial $p_{N}(x)$ in $A(x, t)$ which belongs to the ideal $(1-c x)$, and

6.71

$$
\left\|\sum_{n=0}^{N} a_{n} x^{n}-p_{n}(x)\right\| \rightarrow 0 .
$$

Proof. Recalling 6.33, we see that

where

$$
\sum_{n=0}^{N} a_{n} x^{n}=\sum_{1}^{N} b_{n} x^{n}+\sum_{0}^{N} h_{n} c^{n} x^{n}=\sum h_{n}+\sum b_{n}\left(x^{-1}\right) x^{n}+p_{n}(x)
$$

$$
p_{n}(x)=\sum\left[b_{n} x^{n}-b_{n}\left(x^{-1}\right) x^{n}\right]+\sum h_{n}\left(c^{n} x^{n}-1\right) .
$$

This is clearly a polynomial in $(1-c x)$. The other two terms tend to 0 by 6.52 and $6.62,5.22$, respectively.

The proof of 6.2 is essentially contained in 6.7 , for if 6.71 holds then $\left\|f(x)-p_{n}(x)\right\| \rightarrow 0$.

It is interesting to note that 6.2 is not based on 5.4.

This result (6.2) may be generalized to the case in which $A$ is simply generated by $c$ and a subalgebra $K$. This is intended as a technical term introduced here (ad hoc) in the following way. For each $\lambda$ in the spectrum of $c$ there is a homomorphism $T_{\lambda}$ of bound 1 of $A$ into $K$ such that
6.8
$T_{\lambda}(k a)=k T_{\lambda}(a)$
$(k \in K, a \in A)$,
6.81
$T_{\lambda}(c)=\lambda$ (the scalar),

6.82

Polynomials in c, with coefficients in $K$, are dense in $A$.

7. Some questions. If $c_{1}, c_{2}$ each have an inverse of bound 1 in two superalgebras $B_{1}, B_{2}$, do they each have an inverse of bound 1 in some superalgebra $B$ ? (It cannot be required that $B$ contain $B_{1}, B_{2}$ isometrically.)

There is an analogy between making inverses and solving $c_{1} x_{1}+\cdots$ 
$+c_{n} x_{n}=1\left(c_{1}, \cdots, c_{n}\right.$ given $)$; and the latter problem is related to the removing of ideals. Let the class of nonremovable maximal ideals be called the cortex. It is obviously compact, and [Shilov et al.] contains the Shilov boundary. Can each finite, or even each compact, or even each closed, or even the entire, subset disjoint from the cortex be removed by a single extension? If $A$ is semi-simple, to what extent can this property be carried along?

Turning to our $\S 6$, is $A(t)$ semi-simple when $t=$ the spectral radius of $c$ ? When $t>t_{0}$ ? When $t=t_{0}$ ? How about $A(t)$ in general (3.15)? For monic polynomials, the semi-simplicity problem is surmountable [Hoffman and Arens].

What transcendental equations can be solved in superalgebras? $e^{x}=c$ ? (This equation requires some nonanalytic truncation of the norm if $\log c$ is to be bounded in all cases.)

Appendix, Banach algebras without unit. With completeness, but no unit, we still get that part of 3.1 ending with equation 3.11 i.e., $c$ has an inverse in some superalgebra precisely if it is not a topological zero-divisor. Let us assume 3.12 holds with $t_{0}=1$. We construct the algebra $A_{1}$ of pairs $(\lambda, a)$ (see Loomis, p. 59) and norm it $\|(\lambda, a)\|=|\lambda|+\|a\|$. This has a 1 , and contains $A$. Suppose first that $c$ is a topological zero-divisor in $A_{1}$. Then we would have $a_{n} \in A$, and complex numbers $\lambda_{n}$ with $\left|\lambda_{n}\right|+\left\|a_{n}\right\|=1$ but $c\left(a_{n}+\lambda_{n}\right) \rightarrow 0$. By extracting a subsequence, we achieve $\lambda_{n} \rightarrow \lambda$. Now $\left\|a_{m}-a_{n}\right\| \leqq\left\|c a_{m}-c a_{n}\right\|$ $=\left|\lambda_{m}-\lambda_{n}\right|\|c\|+\epsilon_{m n}$ where $\epsilon_{m n} \rightarrow 0$. Hence $a_{m} \rightarrow a$ for some $a$. Now $|\lambda|+\|a\|=1$ and $c a+\lambda c=0$. If $\lambda$ were 0 , then $0=\|c a\| \geqq\|a\|=1$. Hence we have an element $u$ such that $c u=c$. This $u$ is a 1 for $A$ since $\|b-b u\| \leqq\|c b-c b u\|=\|c b-c b\|$, whence $b=b u$, for every $b$ in $A$. It was pointed out in the proof of 3.1 (Footnote 3 ) that with these assumptions the inverse of $c$ in $A(t)$ would come out not exceeding $\|u\|$.

If, on the other hand, $c$ is not a topological zero divisor in $A_{1}$, then an inverse can be made by appeal to 3.1 (this time $\|1\|=1$ ).

\section{BiBLIOGRAPHY}

1. Richard Arens, Linear topological division algebras, Bull. Amer. Math. Soc. vol. 53 (1947) pp. 623-630.

2. Richard Arens and Kenneth Hoffman, Algebraic extension of normed algebras, Proc. Amer. Math. Soc. vol. 7 (1956) pp. 203-210.

3. Lynn H. Loomis, An introduction to abstract harmonic analysis, New York, van Nostrand, 1953.

4. Charles E. Rickart, The singular elements of a Banach algebra, Duke Math. J. vol. 14 (1947) pp. 1063-1077.

5. I. Gelfand, D. Raikov, and G. Shilov, Commutative normed rings, Uspehi Mat. Nauk N.S. vol. 2 (1946) pp. 48-146.

UNiversity of California,

Los Angeles, Calif. 